\title{
Erratum: European Cities, Municipal Organizations and Diversity
}

DOI 10.1057/978-1-137-52185-9

The affiliation of the author Maria Schiller has been replaced with the below one.

'Maria Schiller, Max Planck Institute for the Study of Religious and Ethnic Diversity, Göttingen, Germany’

The updated original online version for this book can be found at DOI 10.1057/978-1-137-52185-9

(C) The Editor(s) (if applicable) and The Author(s) 2016 